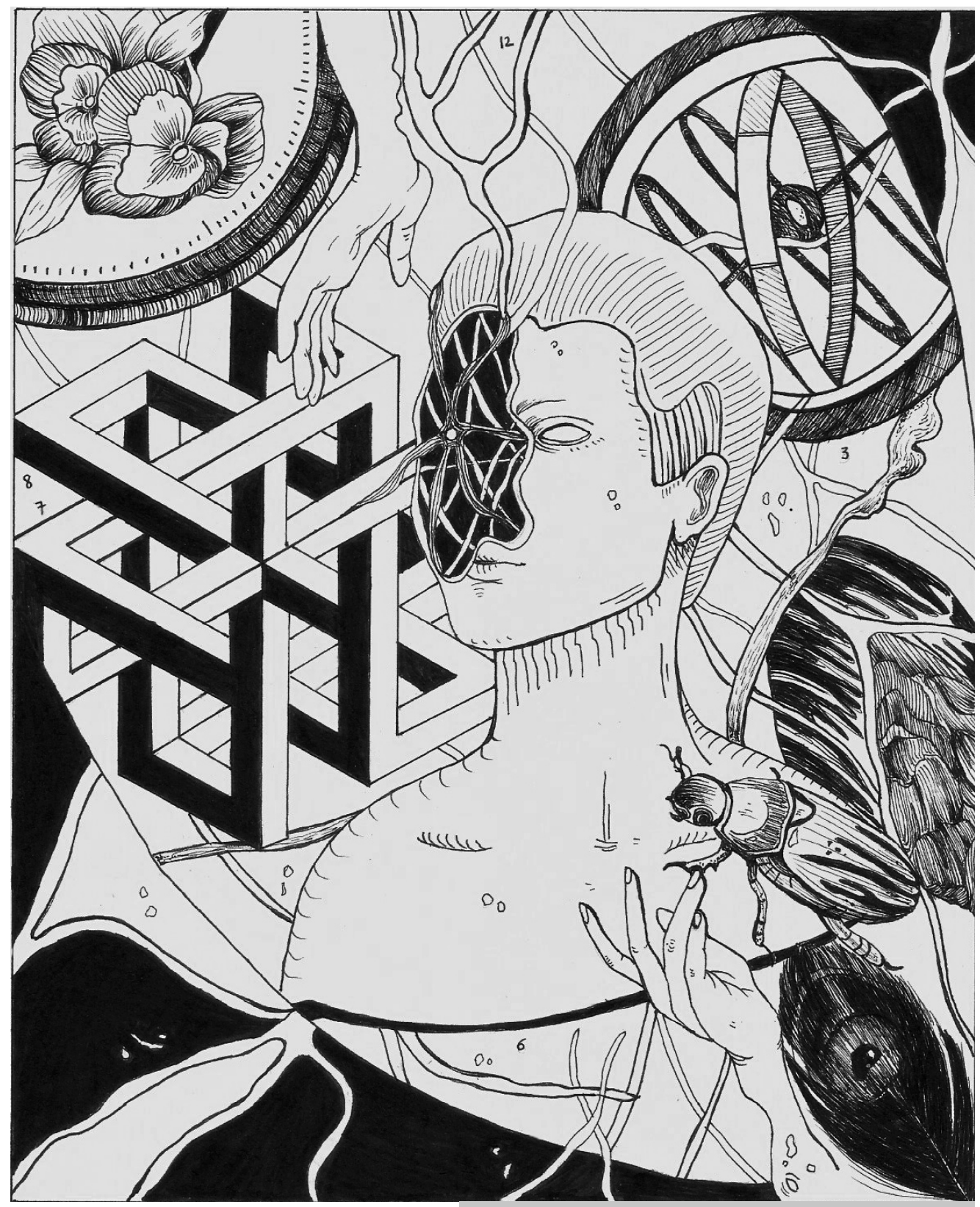

Artista invitada

Elizabeth Rivera Cardona

De la serie Identities

Rapidógrafo en papel aralda

2020

Cortesía Revista Ojo de Pez 


\title{
El giro a la derecha en América Latina. La crueldad y el gobierno de los cuerpos-Otros en la etapa neoliberal del capital ${ }^{*}$
}

\author{
Alejandra del Rocío Bello Urrego (Colombia)**
}

\section{Resumen}

Desde una perspectiva feminista des/poscolonial, este artículo de reflexión responde a la pregunta por la relación entre la reciente llegada al poder de gobiernos de derecha en América Latina, las dinámicas imperiales de gobierno de los cuerpos que han estructurado los Estados-nación de la región y la etapa neoliberal del capital. Se defiende la hipótesis de que el giro ideológico de la región hacia la derecha es una expresión de los modos en que las dinámicas de gobierno de los cuerpos que han definido la normalidad en las naciones latinoamericanas se han actualizado al neoliberalismo. Es esencial decir que ese gobierno se sustenta en una circulación de la crueldad de matriz colonial en la que el sufrimiento se canaliza hacia los cuerpos relegados al lugar de Otro: los no blancos, no binarios, no masculinos, entre otros. Se intenta mostrar cómo los gobiernos del giro han radicalizado la frontera entre la parte de la población que es gobernada bajo lógicas biopolíticas y los Otros sobre quienes la crueldad constituye la principal estrategia de gobierno.

\section{Palabras clave}

Teoría Feminista; Teoría Decolonial; Paraestado; Necropolítica; Derecha Política; América Latina.

\footnotetext{
* Este artículo se deriva del proyecto Cuerpos femeninos y poder en el pacifico colombiano: El caso de la Asociación de Mujeres Ébano, del Departamento de Lenguas y Cultura, Universidad de los Andes, gracias a una beca de Colciencias. En la elaboración del marco de referencia conceptual de este proyecto se dio continuidad a los desarrollos teóricos propuestos en la tesis La gestión moderna del sufrimiento: genealogía del cuerpo sufriente en Colombia, bajo la modalidad de co-tutela entre la Universidad Paris 8 y la Universidad de Brasilia.

** Politóloga. Especialista y magíster en Derechos Humanos y Derecho Internacional Humanitario. Especialista y doctora en Bioética. Doctora en Género y Ciencia Política. Profesora e investigadora de la Universidad del Tolima, Colombia. Correo electrónico: adrbellou@ut.edu.co - Orcid: 0000-00017530-2853 - Google Scholar: https://scholar.google.es/citations?hl=es\&user=ee8MC3AAAAAJ
} 


\title{
Cómo citar este artículo
}

Bello Urrego, Alejandra del Rocío. (2021). El giro a la derecha en América Latina. La crueldad y el gobierno de los cuerpos-Otros en la etapa neoliberal del capital. Estudios Políticos (Universidad de Antioquia), 60, pp. 118-143. DOI: 10.17533/ udea.espo.n60a06

\section{The Turn to the Right in Latin America. Cruelty and the Government of the Bodies-Others in the Neoliberal Stage of the Capital}

\begin{abstract}
This article reflection seeks to answer, from a feminist de/postcolonial perspective, the question of the relationship between the recent arrival to power of right-wing governments in Latin America; the imperial dynamics of the government of the bodies that have structured the Nation-States of the region; the neoliberal stage of the capital, and what is conceptualized here as a patriarchal-colonial channeling of cruelty. The article defends that the region's ideological turn to the right is an expression of the ways in which the dynamics of the government of the bodies that have defined normality in Latin American nations have been updated to neoliberalism. It is important to assert that this government is based on a circulation of the cruelty of colonial-matrix in which suffering is channeled towards bodies relegated to the place of the Other: non-white, non-binary, non-male, etc. We will try to show how the governments of the turn have radicalized the border between the part of the population that is governed under biopolitical logics, and the Others over whom cruelty constitutes the main strategy of the government.
\end{abstract}

\section{Keywords}

Feminist Theory; Decolonial Theory; Parastate; Necropolitics; Political Right; Latin America. 


\section{Introducción}

Este artículo de reflexión teórica aborda el fenómeno de la reciente llegada al poder de gobiernos de derecha a diferentes países de América Latina —en adelante, el giro—, ${ }^{1}$ analizándolo desde uno de sus efectos: el sufrimiento que genera para las poblaciones que históricamente han constituido los Otros $^{2}$ de estas naciones: indígenas, negras, mujeres y lesbianas, gais, bisexuales, transexuales, intersexuales, queer y otrxs $(\mathrm{LGBTIQ}+)$, entre otros.

La hipótesis es que los actuales gobiernos de derecha en la región son una expresión de cómo las dinámicas de gobierno de los cuerpos, que han definido la normalidad en los Estados-nación latinoamericanos desde su génesis, se han actualizado en la etapa neoliberal del capital. Estas dinámicas se sustentan en una circulación de la crueldad de matriz colonial en la que el sufrimiento se canaliza hacia los cuerpos-Otros: no-blancos, no-binarios, no-masculinos, entre otros.

Se entiende por etapa neoliberal de acumulación de capital que comienza con el desmonte de los Estados de bienestar en la década

[120] de 1970 y que se consolida con la transformación de la mayoría de las institucionalidades estatales del mundo bajo los preceptos del Consenso de Washington. En este marco temporal, el neoliberalismo es un discurso hegemónico con efectos sobre las prácticas político-económicas de los Estados y los modos en que existimos en el mundo social (Harvey, 2008). La principal característica de este discurso es promover la radicalización del proceso de acumulación y concentración de riquezas en unos pocos por medio de la desposesión de las mayorías. En una perspectiva foucaultiana, más que un discurso, el neoliberalismo es una racionalidad gubernamental, una grilla de ejercicio del poder que tiene como blanco

\footnotetext{
${ }^{1}$ El giro hace referencia a los gobiernos que llegaron al poder en América Latina en un contexto internacional marcado por el ascenso al poder de Donald Trump en Estados Unidos y de partidos de derecha a los gobiernos europeos. Un contexto regional marcado por el desgaste de los gobiernos de izquierda y la llegada a la Presidencia de Brasil del líder de extrema derecha Jair Bolsonaro.

${ }^{2}$ El Otro es un concepto a partir de los trabajos de la antropóloga Rita Segato (2007) para referirse a la frontera entre las poblaciones mestizas creadas por las políticas de construcción de nación principalmente operadas en la primera mitad del siglo $\mathrm{xx}-\mathrm{y}$ los Otros, entendidos como el conjunto de personas excluidas de este proceso.
} 
principal a la población y que implica un gobierno de las conductas que incita a convertirse en empresario de sí mismo (Foucault, 2008; Saidel, 2018). En ambas perspectivas, el cuerpo está fuera del foco de análisis y el colonialismo está en un segundo plano; por el contrario, desde un prisma de análisis descolonial fanoniano, el cuerpo y su producción, por medio de la violencia, son lo que constituyen los ejes de análisis de la expresión neoliberal de una forma de poder cuya génesis es colonial (Fanon, 2009).

Desde una interpretación feminista materialista descolonial (Dorlin, 2009; Federici, 2018; Lugones, 2008), la ontología del capitalismo es la construcción del cuerpo del animal humano por desposesión: unos cuerpos son más humanos en la medida en que a otros les es negada la posibilidad de devenir humanos, siendo esta acumulación por desposesión de humanidad, que se da en el colonialismo, la condición primera de existencia del capitalismo. Esto implica que la gubernamentalidad neoliberal está inserta en la genealogía del colonialismo (Saidel, 2018), de modo que no se entiende en un sentido puramente foucaultiano como el gobierno de las conductas, ni teniendo a la población de blanco principal, sino como el conjunto amplio y heterogéneo de condiciones para el ejercicio de una forma especifica de poder. Esta forma depende de la jerarquización de la vida, tomando como norma a lo masculino blanco y de la administración de la violencia, el sufrimiento y la muerte. En este sentido, autores como Sayak Valencia (2016) y Achille Mbembe (2006) han explorado la violencia y la muerte desde la perspectiva de cómo estas se transforman en objeto de gobierno. En el caso de Mbembe, este fenómeno fue conceptualizado como necropolítica, y se refiere a la exploración del gobierno del sufrimiento y de la crueldad como mecanismos de gubernamentalidad.

La emergencia de las emociones — como el sufrimiento- y de su relación con la construcción de los cuerpos viene replanteando muchas de las ideas asumidas como dadas sobre el estudio de las relaciones entre poder y cultura (Moraña y Sánchez, 2012). Este artículo se inscribe en los estudios culturales feministas, que desde finales de la década de 1990 vienen posicionando los afectos y el embodiment como categorías que movilizan una apuesta radical para entender y transformar lo político y la cultura, adoptando la propuesta de Sara Ahmed (2014) en lo que concierne a entender la emoción desde la pregunta por cómo esta se materializa en cuerpos individuales y colectivos. 
El sufrimiento es aquí abordado como un marco ${ }^{3}$ histórica y culturalmente contingente para hacer inteligible el cuerpo. Las preguntas por si la gente en efecto sufrió o si desde su perspectiva su experiencia puede hacerse inteligible como sufrimiento o no quedan fuera de este análisis. La pregunta es por cómo una circulación específica del sufrimiento construye cuerpo y constituye una condición para el ejercicio del poder.

Tomando como referencias las conceptualizaciones de las formas modernas del poder aportadas por las corrientes feministas descoloniales, poscoloniales y el black feminism, se entiende que los procesos coloniales que construyeron el globo dieron como resultado la división de la población mundial en términos superior-inferior a partir de la imbricación de la clasificación racial y sexual de los cuerpos (Dorlin, 2008; 2009). Desde esta interpretación feminista de-poscolonial, la zona del no-ser se entiende constituida por los cuerpos que cargan con el signo racial-sexual de los grupos vencidos en las guerras de conquistas (Segato, 2015; 2016a). Estos cuerpos son definidos como Otros a partir del arquetipo de hombre-blanco posicionado como la norma que define el grado de humanidad o inhumanidad.

Dicho esto, el objetivo de este texto es analizar la relación entre el giro [122] a la derecha en las naciones latinoamericanas, las dinámicas imperiales de gobierno de los cuerpos-Otros, la etapa neoliberal del capital y lo que aquí se califica como canalización patriarcal-colonial de la crueldad.

Siguiendo la tradición de la escuela de Frankfort, una temática propia a las ciencias sociales es aquí abordada desde una puesta en cuestión de las metodologías habituales en este campo. No obstante, a diferencia de esta escuela, la crítica no es de orden filosófico en sentido estricto, sino que resulta de una reflexión desde las teorías feministas y descoloniales. Aquí se abraza la intuición en el sentido dado por Aimé Cesaire, Gloria Anzaldua y Audre Lorde. Este encuadre permite que desde las perspectivas intelectuales no hegemónicas se encuentre un lugar desde referentes, emociones y formas que se ajustan al rescate de la propia voz, así como que se pueda conectar la reflexión con contextos y pugnas concretas. Esto no implica, de ninguna

\footnotetext{
${ }^{3}$ La noción de marco se utiliza en este artículo tomando como referencia a Judith Butler (2017), entendidos como un sistema de edición de la realidad del que emergen fronteras, inclusiones y exclusiones siempre cargadas políticamente.
} 
manera, que la experiencia valide por sí sola la riguridad (Scott, 2001), de la misma manera que seguir un método tampoco se traduce en una garantía de la calidad de una investigación (Bentouhami, 2014). La crítica al fetichismo del método - que hace parte de modos de pensar descoloniales como los de Franz Fanon, Fausto Reinaga o Gloria Anzaldúa - es la que se adopta aquí. En esta perspectiva, se invita al lector a discutir con esta investigación de corte teórico, teniendo en cuenta que su propósito no es forzar un acuerdo, sino iniciar una conversación proponiendo un punto de vista y exponiendo transparentemente el modo en que esta perspectiva fue constituida.

En lo que respecta a la organización del texto, de manera coherente a una apuesta de pensamiento feminista descolonial, la aproximación teórica y el contexto histórico de la problemática se desarrollan a través de un caso paradigmático y no desde la abstracción: este caso es el análisis de las relaciones entre la crueldad contra los cuerpos femeninos y las conquistas territoriales del Caribe colombiano por ejércitos paramilitares. La razón detrás de esta elección es que este caso permite ilustrar la idea de que los cuerpos relegados a la zona del no-ser en los procesos coloniales que constituyeron a América Latina fueron los mismos que fueron marginados de la comunidad connacional en los procesos de construcción de nación a inicios del siglo $x x$, y son también quienes devinieron objeto de gobierno a través de la exposición directa a la crueldad en la etapa neoliberal del capital. Una vez establecida esta relación entre la génesis colonial del gobierno de los Otros y la crueldad como estrategia de ejercicio del poder se defiende la idea central de este artículo: los gobiernos del giro en América Latina radicalizaron la división entre la zona del ser y del no-ser que ya era estructural a las naciones de la región.

\section{El caso de la construcción del cuerpo femenino en el Caribe colombiano por parte de las Autodefensas Unidas de Colombia (AUC)}

La región Caribe se compone de siete departamentos: La Guajira, Magdalena, Cesar, Atlántico, Bolívar, Sucre y Córdoba. Al estar sobre el mar Caribe en el océano Atlántico, pero también cerca del océano Pacifico, próximo a las Antillas y Centroamérica, y ser una zona de frontera con Venezuela y Panamá, esta región tiene una geolocalización estratégica desde el punto de vista del funcionamiento del mercado ilegal, especialmente para el narcotráfico 
y el contrabando de armas, electrodomésticos, gasolina, licor, ropa, alimento y precursores químicos (Villarraga, 2014, p. 41). Adicionalmente, es una zona históricamente dejada al margen de la acción del Estado colombiano. De hecho, la mayoría de los habitantes de esta región no fueron objeto de las políticas higienistas adelantadas por el Estado en la primera mitad del siglo xx. En aquella época las estrategias biopolíticas ${ }^{4}$ enfocadas en constituir la población nacional se concentraron en los principales asentamientos urbanos (Noguera, 2003) y esto radicalizó la brecha entre los habitantes higienizados, cuya vida adquirió un valor para el capital —como mano de obra y consumidor-, y los habitantes rurales alejados de los grandes centros urbanos.

La geografía accidentada y la falta de intereses por parte de los gobiernos centrales constituyeron un caldo de cultivo para que en la década de 1970 la región empezara a tener un rol central en el narcotráfico y a contar con la presencia de numerosos actores armados. Además, la altísima concentración de la tierra ha contribuido a que la economía de la población sin tierra se caracterice por la informalidad, el desempleo y la pobreza (Villarraga, 2014, p. 43), y la acción estatal para corregir esta situación ha sido casi inexistente (García y Ojeda, 2018).

[124 ] La eficiencia con que el mercado ilegal ha integrado el territorio caribeño y la falta de presencia estatal han expuesto a estas poblaciones a ser gobernadas por paraestados, cuyas normas operan sin los límites que aplican para las instituciones oficiales: la ley, los derechos humanos, la invulnerabilidad de la vida, la dignidad humana, entre otros. Así, estas vidas son dejadas al margen de las clásicas alianzas entre el poder sapiente, las instituciones modernas y el Estado, haciendo que los cuerpos no sean moldeados bajo estrategias biopolíticas clásicas como las escuelas, el saber médico, entre otros.

El filósofo Achile Mbembe (2006) propone el concepto necropolítica para referirse a las técnicas de gobierno de estas poblaciones dejadas al margen. Según él, el ejercicio del poder se da en la acción de decidir quién debe morir a partir de un criterio de rentabilidad económica. En esta perspectiva, la lógica amigo-enemigo permite producir un estado de excepción permanente que justifica la administración de la muerte.

\footnotetext{
${ }^{4}$ Biopolítica se entiende como una política de la vida y hacia la vida en la que se convierte en objeto del poder sapiente, haciendo del cuerpo el escenario en que este poder se transforma en materia (Foucault, 2007).
} 
Esta propuesta conceptual permite entender las formas del poder que se expresan en la conquista del Caribe colombiano por las AUC en la década de 1990. Por ejemplo, el ejercicio del poder en el Caribe se inscribe en una lógica amigo (la nación)-enemigo (el insurgente) que justifica un estado de excepción permanente en el cual la administración de la muerte desempeña un rol central. Sin embargo, en el caso colombiano, el ejercicio del terror contra aquellos que en el proceso de constitución de la nación colombiana estuvieron relegadas al lugar de los Otros parece más ejercer y manifestar el poder sobre la vida que administrar la muerte.

Esta división entre el escenario de lo biopolítico y de lo necropolítico resuena con la división entre lo humano y lo subhumano, descrita como constitutiva de la organización colonial del mundo por Fanon (2009) en su conceptualización de la división entre el ser y el no-ser. La acción de hacer de un cuerpo el objeto de técnicas biopolíticas o necropolíticas es una estrategia para transformar en carne relaciones de dominación, y esta estrategia ubica el cuerpo en relación con la frontera entre la zona del ser y la del no-ser.

En la perspectiva de Rita Segato (2007), las lógicas coloniales del poder se introdujeron en los Estados-nación de la región a través de la manutención de una frontera entre quienes constituyen la Nación y los Otros, a quienes aun habitando el territorio nacional no se les ha reconocido una ciudadanía plena. Esta lógica de inclusión y exclusión es constitutiva de los Estadonación latinoamericanos. Se trata de una zona del no-ser constitutiva a estas formaciones nacionales y la inserción a la economía global del lado ilegal del mercado y el ser un territorio históricamente al margen de la acción estatal hacen de la región Caribe un ejemplo paradigmático del tipo de territorio y de población que constituyen la zona del no-ser.

El gobierno parainstitucional de ciertos territorios del Caribe colombiano estuvo acompañado de la cooptación de los espacios públicos y de los dineros públicos, así como de la regulación de la vida cotidiana de los habitantes de los territorios invadidos (CNMH, 2011). Si bien este último elemento tal vez constituya uno de los efectos menos estudiados del conflicto colombiano, puede ser el escenario en el que se desempeñan algunas de sus repercusiones más profundas. Por ejemplo, el disciplinamiento de los cuerpos femeninos que se dispuso en esa regulación de la vida cotidiana buscó introducir cambios profundos en las formas de vida de las personas, una regulación de género capaz de afectar la relación de las personas, hombres y mujeres con su propio 
cuerpo y el de los otros, y esos cambios son capaces de persistir más allá de la presencia del grupo armado.

En los dos informes de la Comisión de Memoria Histórica aquí analizados se reportaron los siguientes perfiles en las víctimas: indiscriminado - por ser mujer-, transitivo, estigmatizado, emblemático-representativo y transgresor $(\mathrm{CNMH}, 2011$, capítulo 3). El perfil indiscriminado se ilustra en el caso del concurso de belleza organizado por las AUC en que se obligó a participar a las mujeres designadas por el grupo y a la comunidad a asistir. Las participantes, la mayor parte menores de edad, fueron violadas. Este caso, muestra cómo el gobierno de los cuerpos implicó una radicalización de la diferencia sexual en que las mujeres fueron relegadas al rol de objetos sexuales $(\mathrm{CNMH}, 2011 ; 2015)$.

En el perfil transitivo se clasificó la violencia perpetrada contra una mujer acusada de tener un vínculo sentimental o familiar con un guerrillero, mientras que en el estigmatizado se clasificó la perpetrada contra mujeres acusadas de pertenecer a la guerrilla. En cuanto al perfil transgresor, un ejemplo de quienes fueron objeto de crueldad por encajar en este es el caso de un combate de boxeo organizado por las AUC en que obligaron

[126] a pelear frente a todo el pueblo al «bando» de los homosexuales contra el de las mujeres chismosas $(\mathrm{CNMH}, 2011 ; 2015)$. Esto constituye un ejemplo de cómo se implantó el modelo de la heteronormatividad obligatoria, cuya estrategia fue la exposición a la crueldad extrema o la aniquilación de todo cuerpo que lo desafiara, ya fuera desde habitar de forma no binaria el cuerpo o desde el desafío a los roles de género hegemónicos.

Sobre el perfil emblemático-representativo, es relatado el caso de tres mujeres cuyo cuero cabelludo fue arrancado en público $(\mathrm{CNMH}, 2011$; 2015). Según el testimonio de una de ellas, embarazada de dos meses en el momento de los hechos, las AUC le arrancaron el cuero cabelludo en público después de haberla desnudado mientras le decían «perra, hija de puta, tu vida no vale nada y tu mereces la muerte». Dos días antes, la misma mujer y su prima habían sido secuestradas por paramilitares quienes les decían «hijas de puta, ustedes existen para cuidar a los niños y no para ir a fiestas» (CNMH, 2011, pp. 70-71). Este caso muestra cómo el ejercicio del poder buscó negar el reconocimiento de la humanidad a los cuerpos femeninos que no encarnan un modelo de feminidad definido por roles domésticos y de reproducción. 
Muchas de las víctimas responden a los dos últimos perfiles, es decir, eran mujeres que ejercían roles clave en la cohesión comunitaria o roles de autoridad, mujeres que no encajaban en el modelo de feminidad doméstica y que, en términos generales, no encajaban en una norma de género específica caracterizada por la división binaria, patriarcal, heterosexual y jerárquica de los cuerpos. Las mujeres líderes y chismosas no solo fueron objeto de la crueldad debido a ser mujeres, sino también por los vínculos comunitarios que existen a través de ellas. Aniquilar los cuerpos de quienes tejen esos vínculos es un ataque a la posibilidad de existencia misma de la vida comunitaria.

Los disidentes de la norma de género fueron transformados en cuerpos desechables cuyo uso se restringió a enseñar a los habitantes de los territorios invadidos las normas que delimitan las posibilidades de existencia. No solo se buscó naturalizar ciertos niveles de crueldad, como siendo propios a la existencia de cuerpos disidentes, sino que además se buscó banalizar las acciones tendientes a negarles la posibilidad de existir. Estos cuerpos fueron aniquilados para aniquilar la posibilidad de estar en el mundo que estaba encarnada en ellos (Centro Nacional de Memoria Histórica, 2015).

Las receptoras del mensaje enviado a través de la crueldad fueron todas las mujeres y sus comunidades, y no solo las afectadas directamente. En esa medida, esta violencia constituyó un ejercicio pedagógico en la medida en que enseñó cuáles maneras de ser mujeres son posibles y cuáles no. Esto deja ver que el objeto de esta crueldad fue destruir formas de existencia susceptibles de desafiar los intereses representados por las AUC, la consolidación del proyecto histórico del capital.

\section{Hacer sufrir, la zona del no-ser y el giro a la derecha en América Latina}

«Los derechos humanos son para los humanos derechos ». Pronunciamiento del alcalde de Tijuana, México, Juan Manuel Gastelum, respecto a la caravana migrante en 2018 (Camhaji, 2018, noviembre 17).

Los gobiernos de Iván Duque en Colombia (2018-2022), Jair Bolsonaro en Brasil (2019-2023), Sebastián Piñera en Chile (2018-2022), Mauricio Macri en Argentina (2015-2019), Enrique Peña Nieto en México (2012-2018), Lenin Moreno en Ecuador (2017-2021) y Pedro Pablo Kuczynski en Perú (2016-2018) 
tienen en común los siguientes elementos: el apoyo de las Iglesias cristianas fue determinante en su elección; al menos en los casos de Chile, Argentina, Perú, Colombia y Brasil, la satanización de las opciones de izquierda, utilizando a Venezuela como símbolo de debacle, fue un elemento clave en sus campañas o discursos; la defensa de la familia tradicional y la lucha anticorrupción fueron valores estandarte en sus campañas; han contado con el apoyo de los grandes sectores económicos con intereses en la tierra y tienen posturas favorables a EE. UU. (Mariano y Gerardi, 2019; Tauli-Corpuz, 2018, agosto).

En esta sección se aborda el caso de la defensa de la «vida» y la «familia» como valores de estos gobiernos, y luego los rasgos generales de las políticas de tierras defendidas por estos mandatarios. Estos escenarios son transversales a todos los casos e ilustran cómo estos gobiernos participan de una canalización específica de la crueldad.

\subsection{En nombre de la vida}

Multiplicaré los sufrimientos de tus embarazos; darás a luz a tus hijos con dolor. Sentirás atracción por tu marido, y él te dominará.

(Génesis 3:16).

Desde la década de 1980 las Iglesias evangélicas vienen ganando adeptos en los sectores históricamente dejados al margen de la acción estatal: clases populares urbanas, sectores rurales y comunidades indígenas y negras (Mariano y Gerardi, 2019). En la última década su capacidad de injerencia en política se hizo evidente, particularmente en las elecciones de $2018 .{ }^{5} \mathrm{Su}$ capital político radica en la defensa de la vida y la familia tradicional, y la lucha contra la ideología de género (Mariano y Gerardi, 2019). Estos principios se tradujeron en exigencias políticas concretas: prohibición del aborto y del matrimonio igualitario, posibilidad de injerencia en temas de educación

\footnotetext{
${ }^{5}$ Veáse sobre Duque Ariel Ávila (2018, mayo 14), Daniela Amaya (2018, mayo 11), La W Radio (2018, mayo 9); sobre Bolsonaro véase Rodolfo Borges (2018, octubre 31), Estadão Conteúdo (2018, octubre $\left.1 .^{\circ}\right)$; France 24 (2018, octubre 21); sobre Piñera véase El Desconcierto (2018, noviembre 14), Prensa Presidencial (2018, octubre 31); sobre Macri véase Evangélico Digital (2018, septiembre 19), Infobae (2017, septiembre 5), Mundo Cristiano (2018, septiembre 18), Noticias (2018, octubre 8), Salgado, (2018, octubre 17); sobre Peña Nieto véase Carlos Lara (2017, febrero 16); sobre Moreno véase Agencia EFE (2018, noviembre 27); Ivan Witker (2018, octubre 15); sobre Kuczynski y Vizcarra véase Martín Hidalgo (2017, noviembre 12), Gonzalo Ruiz (2016, abril 12).
} 
sexual en colegios y oposición a toda propuesta proveniente de sectores feministas y a cualquier iniciativa que implique reconocimiento de los cuerpos y existencias disidentes del género.

La gestión estatal de los úteros que se disputa en el control de la natalidad es una medida concreta en la que la dominación se convierte en carne. Trabajos como los de Elsa Dorlin $(2008 ; 2009)$ o de François Vergès han mostrado que en los Estados-nación modernos la racialización de la población se ha disputado a través de la gestión de los vientres, imponiendo o impidiendo la maternidad. Han dejado en evidencia que para los Estados modernos la imposición de la maternidad sobre las mujeres blancas y la negación de esta a las no-blancas ha sido un eje de la gubernamentalidad nacional e imperial. La eugenesia no ha sido ajena a la época actual, con casos como las esterilizaciones forzadas en el Perú fujimorista (Mantilla, 2001). Sin embargo, este no es un elemento de los discursos de los Estados pos reformas neoliberales, sino de la propia consolidación de los Estadosnación latinoamericanos durante la primera mitad del siglo xx.

Las Iglesias evangélicas están en contra de cualquier forma de eugenismo, de hecho, la oposición al aborto defendida desde estas plataformas impone la maternidad a todas las mujeres. Sin embargo, asuntos como la concentración de la casi totalidad de muertes por abortos ilegales, los feminicidios en gestantes - principal causa de muerte de mujeres en embarazo en el mundo- y la mortalidad materna en mujeres no blancas, pobres o rurales (CLAM, 2011, septiembre 21; OMS, 2019, septiembre 19; Blum y Qureshi, 2011; Costa, Ribas, Amorim y Santos, 2002; Horon y Cheng, 2001), no son prioritarios en sus agendas. ${ }^{6}$

Por otro lado, si bien la familia y la vida se presentan como valores supremos, el equivalente masculino del aborto, el abandono paterno, no es un punto prioritario. Este tipo de imposición es un mecanismo efectivo para mantener a las mujeres atrapadas en las expectativas que una sociedad pone sobre ellas, siendo la maternidad impuesta ${ }^{7}$ un mecanismo efectivo para mantener a las mujeres atrapadas en cuerpos forjados a imagen y semejanza de los intereses del capitalismo colonial y patriarcal (Saadawi, 2010). En esta

\footnotetext{
${ }^{6}$ Fenómenos que constituyen una forma de eugenismo, en la medida en que seleccionan con criterios en los que la raza, el sexo y la clase están imbricados a cuáles cuerpos el Estado garantiza condiciones para su reproducción y a cuáles no.

${ }^{7}$ Junto con el imperativo de belleza y feminidad (Sa'dāwī y Horst, 2010).
} 
perspectiva, la maternidad es definida como una cárcel de la cual el hombre puede escapar, pero la mujer no (Sanchís, 2017, marzo 30).

De manera complementaria, la negación de derechos a la población LGBTIQ+ persigue la posibilidad de habitar el mundo desde cuerpos que rompen con la prisión corporal del género. Esto segrega de la vida ciudadana a los cuerpos que encarnan disidencias a la heterosexualidad — gai, lesbiana, pansexual, asexual, entre otros - o a la división binaria de los cuerpos intersexual, transexual, entre otros-.

Se observa en las agendas evangélicas una regulación de género basada en la división binaria y jerárquica de los cuerpos, la organización patriarcal y colonial de lo social, y la heterosexualidad obligatoria. Nótese que esta misma regulación fue la que las AUC buscaron implantar en Colombia. Esta similitud no es una coincidencia, pues este tipo de regulación siempre ha sido un eje fundamental del capitalismo, al menos en dos aspectos: «La regulación del género y el disciplinamiento de los cuerpos femeninos aporta el trabajo no remunerado de cuidado y reproducción de la vida sobre el que se sustenta el trabajo asalariado» (Federici, 2018). ${ }^{8}$

[130 ] La clasificación racial de la población mundial imbricada a su clasificación desde una norma eurocéntrica del género fue un eje de la expansión colonial de Europa. El género constituyó la punta de lanza de la destrucción de los mundos invadidos y de la posterior integración del pueblo colonizado al sistema-mundo-colonial, desde la construcción de América en el siglo xvI (Lugones, 2008; Ochoa, 2014), pasando por la expansión europea en África en el siglo xIx (Oyěwùmí, 1997) y hasta las expansiones del neoliberalismo en épocas contemporáneas (Segato, 2014).

La regulación de género defendida por las agendas evangélicas y adoptada por los gobiernos del giro se inserta en estas dinámicas. Medidas como la prohibición del aborto o la negación de derechos civiles contribuyen fuertemente a la estandarización de valores y normas que regulan el cuerpo. Esto reifica el lugar de subalternidad de los sujetos con útero, así como de cualquiera que desafíe el binarismo y la heterosexualidad.

\footnotetext{
${ }^{8}$ Además de ser una de las tesis centrales de Silvia Federici, este es uno de los principales postulados del feminismo materialista francés. Al respecto véase Anni Bidet-Mordrel et al. (2016) y Oriane Petteni y Sophie Wustefeld (2016).
} 
Esto tiene repercusiones muy concretas en la cotidianidad de las personas: el aborto inseguro hace parte de las cinco causas que en su conjunto constituyen $75 \%$ de la mortalidad materna (OMS, 2019, septiembre 19). En los últimos veinte años el número de abortos inducidos en América Latina aumentó de 4.4 millones en el periodo 1990-1994 a 6.5 millones en el periodo 2010-2014 (Guttmacher Institute, 2018, marzo). La región es al mismo tiempo una de las que tiene la mayor mortalidad del mundo por abortos inseguros, con una tasa de 30 muertes o más por cada 1000 mujeres entre 15 y 44 años, y una de las que cuenta con las leyes más restrictivas al respecto (Gómez, 2016).

Estas restricciones afectan desproporcionadamente a mujeres pobres, indígenas y negras, quienes son la que más se ven obligadas a recurrir a abortos inseguros (Sieder, Beltrán, Machado y Peñas, 2019, pp. 240-241). Según la Organización Panamericana de la Salud y la Federación Internacional de Ginecología y Obstetricia, las medidas más efectivas para reducir estas cifran son la garantía de acceso a procedimientos médicos seguros, a anticonceptivos y a educación sexual (Gómez, Urquijo y Villarreal, 2010; Shaw, 2010). Y todas estas son medidas que van en contravía de lo que los grupos evangélicos han exigido a los gobiernos del giro a cambio de su respaldo.

En América Latina se presentan altos índices de violencia contra personas de la comunidad LGBTIQ+. La Comisión Interamericana de Derechos Humanos estableció que en la región la violencia física contra esta comunidad se caracteriza por un alto nivel de «ensañamiento y crueldad». Es habitual que «los cuerpos sin vida de personas LGBTIQ+ demuestren que han sido torturados, sus genitales mutilados, sus cuerpos descuartizados y marcados con símbolos» (Comisión Interamericana de Derechos Humanos, 2015, p. 12). El ensañamiento muestra que, más que la muerte, lo que está en juego es el martirio del cuerpo, el cual busca enviar un mensaje a toda la sociedad: desafiar el género tiene como respuesta la exposición a sufrir.

La crueldad no se canaliza de manera uniforme en la comunidad LGBTIQ+, esta se concentra en función de la intersección entre la raza, el sexo, la orientación sexual, la situación migratoria, la edad, el perfil de defensor o defensora de derechos humanos, y la pobreza (Comisión Interamericana de Derechos Humanos, 2015, capítulo 5). Las legislaciones restrictivas y los discursos públicos de los gobiernos son elementos claves en la naturalización 
de la discriminación, la estigmatización y la violencia dirigida hacia esta población (pp. 236-243).

La adopción de las agendas evangélicas en asuntos de género no es una concesión menor por parte de los gobiernos del giro. Lo que allí se juega es un mecanismo concreto de regulación del género y de construcción de los cuerpos. La prohibición del aborto, la oposición a los derechos de la comunidad LGBTIQ + y la lucha contra la «ideología de género» constituyen eslabones clave en una canalización específica del sufrimiento. Los discursos defendidos públicamente y las medidas jurídicas adelantadas por los gobiernos del giro en favor de estas agendas hacen parte de lo que Segato (2016b) califica de pedagogía de la crueldad, es decir, del conjunto de elementos que enseñan, contribuyen y naturalizan la acción de transformar la vida en cosa, en no-humano, en no-ser.

Por lo tanto, los compromisos de los gobiernos del giro con la protección de la «familia» y la «vida» desde una perspectiva evangélica se traducen en naturalizar los sufrimientos de mujeres y disidentes del género, como siendo parte de las acciones necesarias para construir la sociedad deseable. Sufrimientos que circulan en consonancia con la clasificación de las personas

[132 ] desde la imbricación de la raza, la clase y el sexo. En esta medida, estamos frente a cuerpos sacrificiales cuyo sufrimiento - no necesariamente su muerte- no es un efecto colateral, sino el objetivo de esta forma de gobierno.

\subsection{En nombre de la tierra}

«Si la autoridad, serena, firme y con criterio social implica una masacre [de los pueblos indígenas del Cauca colombiano organizados en Minga] es porque [...]».

Senador colombiano Álvaro Uribe justificando una masacre contra de la Minga de los pueblos indígenas del Cauca (Semana, 2019, julio 4).

«El reconocimiento de tierras indígenas es un obstáculo para la agroindustria»: Jair Messias Bolsonaro (Watson, 2018, diciembre 31).

La inclusión de tierras que estaban al margen de la acción estatal al mercado es un rasgo de la etapa neoliberal del capital (Harvey, 2007, pp. 111-124). Es un proceso global de «reconversión» de tierras olvidadas a tierras productivas. Esto significa transformar territorios en bienes capitalizados 
que pasan a ser activos de grandes corporaciones transnacionales del sector de la agroindustria, el petróleo, la minería, la construcción y el turismo, entre otros. Inversiones que generan enormes ganancias como consecuencia de las condiciones favorables garantizadas por los Estados receptores. Por ejemplo, mano de obra disciplinada, barata y desregulada, así como recursos naturales desregulados de fácil explotación (Márquez, 2017, p. 208).

Sin negar la importancia del análisis materialista, es importante recordar que el despojo no es solo un proceso económico, sino, ante todo, un proceso de colonización. La acumulación de las tierras es una parte de un proceso complejo de invasión y reconstrucción de lo invadido bajo los intereses del invasor: un proceso cultural.

En esta sección se analizarán dos rasgos que son comunes a los gobiernos del giro: intensificación de la persecución violenta a líderes sociales que defienden los territorios frente a explotación minera, maderera, agroindustrial, turística, entre otros; e intensificación de procesos que destruyen lo comunitario y la relación comunidad-territorio con participación o anuencia del Estado.

En agosto de 2018 la relatora especial para los derechos indígenas Victoria Tauli-Corpuz (2018, agosto) denunció que «la rápida expansión de proyectos de desarrollo en tierras indígenas sin su consentimiento previo está impulsando un drástico aumento de la violencia y el acoso legal contra los pueblos indígenas». En una carta dirigida a los jefes de gobierno del mundo señaló que $67 \%$ de los 312 defensores de los derechos humanos asesinados en 2017 estaban defendiendo sus tierras y que $80 \%$ de los homicidios ocurrieron en solo cuatro países, tres de los cuales son latinoamericanos: Brasil, Colombia, México y las Filipinas. Según Tauli-Corpuz, la tendencia global es la siguiente: «Siempre se suceden desde el poder campañas de difamación y discursos de odio que caracterizan a los pueblos indígenas como "obstáculos para el desarrollo", o en el peor de los casos, como "terroristas" o "matones" ». Luego sobreviene la criminalización, órdenes de arresto y violencia sobre las comunidades, existiendo una impunidad generalizada para quienes cometen actos de violencia.

Todos los gobiernos del giro han contribuido al fenómeno de la persecución de líderes de la defensa de la tierra. Los casos más extremos son los siguientes: 
En México, el gobierno de Peña Nieto (2012-2018) se posicionó entre los tres primeros del mundo en asesinato de líderes sociales ${ }^{9}$ y como el gobierno más mortífero de la historia del país, con un total de 125000 personas asesinadas (Nájar, 2018, octubre 26). En el caso de los líderes, en su gran mayoría se trataba de indígenas defendiendo sus territorios (Tauli-Corpuz, 2018, agosto), y en cuanto a sus responsables fueron mayoritariamente fuerzas estatales y paraestatales. En este periodo cada año aumentaron considerablemente los asesinatos, siendo 2017 el año con más casos contra defensores de la tierra en la historia del país (Global Witness, 2018, julio 24).

En el Brasil de Luiz Inácio Lula da Silva (2003-2010) y de Dilma Rousseff (2011-2016) la situación de las y los defensores de la tierra no era la mejor por cuenta del peso de las economías extractivistas en los presupuestos estatales. Así, si bien Bolsonaro (2019-2023) no introdujo una dinámica nueva, sí la llevó a extremos sin precedentes. ${ }^{10}$ Organismos como la ONU, Amnistía Internacional y Human Rights Watch han advertido sobre el riesgo inminente de genocidio al que se enfrentan no solo los líderes indígenas, sino pueblos enteros durante este gobierno (Watson, 2018, diciembre 31; Phillips, 2019, marzo 4; Amnistía Internacional, 2018, octubre 28; Prakash, 2019, abril 18).

[134] Las poblaciones negras rurales y urbanas también están fuertemente amenazadas. La política de «carta blanca» para que la policía controle el crimen se ha traducido en asesinatos y torturas — como violación — contra las poblaciones negras (Human Rights Watch, 2019, enero 17).

En Colombia, los asesinatos desmesurados contra líderes sociales comenzaron durante el gobierno de Juan Manuel Santos (2010-2018) y continuaron durante el de Iván Duque (2018-2022), Ilegando a sumar más de 600 líderes desde la firma del Acuerdo de Paz en 2016 hasta hoy. En 2018, el fenómeno aumentó en 51\% respecto a 2017 y las cifras de 2019 muestran que solo en enero hubo 32 casos, lo que indica que durante el gobierno Duque hay una tendencia al aumento (Hurtado, Gutiérrez, Gómez y Barbosa,

\footnotetext{
${ }^{9}$ Cabe recordar que el emblemático caso de los 43 normalistas desaparecidos en Ayotsinapa, estado de Guerrero, ocurrió al inicio de su gobierno.

${ }^{10}$ Por ejemplo, su primera acción de gobierno fue un decreto que pone la tarea de demarcar las tierras indígenas en manos de la ministra de Agricultura Tereza Cristina Correa, la líder de la Unión Democrática Ruralista. Plataforma desde la que se defienden los intereses de ganaderos, mineros y demás sectores de la industria extractivista (Watson, 2019, abril 14).
} 
2019, marzo, p. 2). Además de la cantidad abrumadora, no es extraño que estos crímenes estén marcados por la sevicia, especialmente cuando se trata de mujeres y población LGBTIQ (Centro Nacional de Memoria Histórica, 2015).

En cuanto a sus responsables, en su casi totalidad estos crímenes son perpetrados por fuerzas estatales o paraestatales, y las víctimas son afrocolombianos, indígenas o campesinos (Human Rights Watch, s. f.). La situación es particularmente dramática en Chocó, Nariño y Cauca, territorios historialmente olvidados por el Estado y habitados en su gran mayoría por comunidades afrocolombianas e indígenas (Amnesty International, 2019, abril 18).

Los gobiernos del giro han intensificado los ataques ante la posibilidad de existencia de lo comunitario, principalmente con dos mecanismos: a) leyes que debilitan los derechos y la tenencia colectiva sobre la tierra, medidas que impulsan el «emprendimiento individual», al mismo tiempo que privilegian a los grandes terratenientes, relegando a los Otros al rol de mano de obra; ${ }^{11}$ y b) negar total o parcialmente el derecho a la consulta previa de las comunidades respecto a proyectos económicos en sus territorios (Human Rights Watch, s. f.; Tauli-Corpuz, 2018, agosto). Esto deja a las comunidades sin canales institucionales para oponerse a la llegada de dinámicas económicas, políticas y de violencia que destruyen el tejido comunitario.

En los discursos, leyes o actos de Bolsonaro, Piñera y Macri la intención de «civilizar» a las comunidades indígenas y negras es explícita, y aunque en los de los otros gobiernos no siempre sea tan clara, está igualmente presente, pues la intención se acompaña de medidas jurídicas que ofrecen garantías a inversionistas para explotar la tierra y una amplia tolerancia al ejercicio de la violencia contra quienes se opongan. Otro elemento común es que las garantías ofrecidas para la explotación de la tierra no se acompañan de inversiones estatales en las instituciones cuya ausencia histórica ha sido parte constitutiva de la exclusión de estos territorios y sus habitantes: escuelas, hospitales, saneamiento, urbanismo, entre otros. ${ }^{12}$

\footnotetext{
${ }^{11}$ La Ley CIDRES de Duque en Colombia, la modificación a la ley indígena propuesta por Piñera en Chile, y la modificación en las políticas de demarcación de tierras implementada por Bolsonaro en Brasil son buenos ejemplos.

${ }^{12}$ Los casos del Acuerdo Nacional por la Paz y el Desarrollo de Piñera o la ley CIDRES de Duque son buenos ejemplos.
} 
La confluencia de intereses entre los gobiernos del giro y los sectores de la economía transnacional ligados a la explotación de la tierra ha redundado en un claro retroceso respecto a las concesiones hechas en el contexto del multiculturalismo liberal de la década de 1990, como es el caso de los derechos colectivos sobre la tierra. En efecto, la atracción y protección de inversionistas se traducen en condiciones que exponen a morir y principalmente a sufrir a las mismas poblaciones que fueron dejadas al margen de las estrategias biopolíticas a través de las cuales se consolidaron las naciones de la región a inicios del siglo xx: indígenas, afrodescendientes y campesinas.

La estrategia necropolítica es clara en los casos de asesinato de las personas que se interponen a la explotación de los territorios; sin embargo, la muerte también es un mensaje para quienes quedan vivos, pues indica los límites de las posibilidades de existencia. Este mensaje funciona en cuanto hace sufrir a quienes quedan, disciplinando por este medio sus cuerpos. De este modo, nos encontramos frente a una estrategia de gobierno que se resume en la administración de la crueldad.

\section{A modo de conclusión: la actualización de la zona del no-ser en las naciones latinoamericanas \\ [136 ] en la etapa neoliberal del capital}

"He escuchado historias de otras personas que han muerto, y amigos que me dicen que otros han sido golpeados por otras pandillas [...]. Es frustrante oír esto todos los días y que nadie haga nada al respecto [...]. Vivimos aquí, así que tenemos que lidiar con ello, pero es desgarrador».

Sebastián Urrutia Lutz, hombre gay chileno sobreviviente de ataque callejero (Brochetto, 2017, febrero 27).

«El Gobierno no está respetando nuestros derechos. Está entregando nuestras tierras a las multinacionales para que exploten el oro, el oxígeno, los bosques, la biodiversidad y la madre tierra. Para ellos es una mercancía que intercambian por dinero que luego malgastan». Giovanni Yala, vocero indígena del Consejo Regional Indígena del Cauca, Colombia (Vargas, 2019, marzo 14).

El caso de la conquista del Caribe colombiano por parte de las AUC muestra cómo los cuerpos femeninos fueron transformados en cuerpos sacrificiales en la implantación de un gobierno parainstitucional de la vida. 
Este caso ilustra los modos en que operaba la crueldad como estrategia de gobierno sobre los Otros desde antes de la llegada del giro. De esta forma, estos gobiernos no introdujeron una dinámica nueva, la radicalizaron. Transformaron la crueldad contra mujeres, disidentes de género, indígenas, negros y campesinos en elementos estructurales de los discursos y acciones del Estado; además, al asociar esta canalización específica a la construcción de un proyecto de sociedad — proyecto del cual hacen parte todas las personas que votaron o apoyan a estos mandatarios- contribuyeron a la naturalización de una cultura política que tolera y defiende una circulación patriarcal y colonial del sufrimiento.

La expansión del Estado hacia territorios indígenas, afrodescendientes y campesinos con el fin de explotar la tierra es una dinámica global y propiamente neoliberal que ya estaba presente antes del giro. En este aspecto, la novedad introducida fue la radicalización de la crueldad como estrategia de gobierno respecto a las poblaciones-pueblos que habían sido dejados al margen de las naciones. En estos territorios los mandatarios de derecha dejaron de lado los elementos de política social que, en algunos casos, acompañaron las economías extractivistas durante los gobiernos de izquierda y radicalizaron el elemento represor.

Los gobiernos del giro han radicalizado la frontera entre el ser y el noser al naturalizar una canalización de la crueldad en la que la dosificación depende de la lejanía de un cuerpo respecto al arquetipo de hombre-blanco. Mientras que unos cuerpos son más el objeto de estrategias de gobierno biopolíticas, otros lo son más de la administración de la crueldad. En esta perspectiva, estos gobiernos se ubican como actores claves en la actualización de las modalidades de gobierno de los cuerpos a la etapa neoliberal del capital.

\section{Referencias bibliográficas}

1. Agencia EFE. (2018, noviembre 27). Lenín Moreno lidera un encuentro inédito con los líderes religiosos de Ecuador. El Economista. https://ecodiario.eleconomista. es/espana/noticias/9547999/11/18/Lenin-Moreno-lidera-un-encuentro-inedito-conlos-lideres-religiosos-de-Ecuador.html

2. Ahmed, Sara. (2014). The Cultural Politics of Emotion. Edinburgh: Edinburgh University.

3. Amaya, Daniela. (2018, mayo 11). Con el Mira, se confirma que Duque es el elegido de los cristianos. La Silla Vacía. https://lasillavacia.com/con-el-mira-seconfirma-que-duque-es-el-elegido-de-los-cristianos-66048 
4. Amnesty International. (2019, abril 18). Urgent Action. Thousands in Need of Urgent Protection. https://www.amnestyusa.org/urgent-actions/urgent-actionthousands-in-need-of-urgent-protection-colombia-ua-52-19/

5. Amnistía Internacional. (2018, octubre 28). Brasil: El discurso tóxico no debe convertirse en política oficial. https:/www.amnesty.org/es/latest/news/2018/10/braziltoxic-speech-must-not-become-government-policy/

6. Ávila, Ariel. (2018, mayo 14). La caverna de Iván Duque Los líderes de la mayoría de las iglesias cristianas apoyan la campaña del candidato uribista. El País. https://elpais.com/internacional/2018/05/15/colombia/1526401037_967975.html

7. Bentouhami, Hourya. (2014). Fanon, critique du «fétichisme méthodologique». Entretien avec Lewis Gordon. Actuel Marx, 55 (1), pp. 49-59. https://doi.org/10.3917/ amx.055.0049

8. Bidet-Mordrel, Anni; Galerand, Elsa \& Kergoat, Danièle. (2016). Analyse critique et féminismes matérialistes. Travail, sexualité(s), culture. Cahiers du Genre, 4 (3), pp. 5-27. https://doi.org/10.3917/cdge.hs04.0005

9. Blum, Robert \& Qureshi, Farah. (2011). Morbidity and Mortality among Adolescents and Young Adults in the United States. Johns Hopkins Bloomberg School of Public Health. https://www.jhsph.edu/research/centers-and-institutes/center-foradolescent-health/_images/_pre-redesign/az/US\%20Fact\%20Sheet_FINAL.pdf

10. Borges, Rodolfo. (2018, octubre 31). La agenda evangélica se eleva al poder con Bolsonaro. El País. https://elpais.com/internacional/2018/10/30/ [138] america/1540915888_923008.html

11. Brochetto, Marilia. (2017, febrero 27). La compleja realidad de ser gay en América Latina. CNN. https://cnnespanol.cnn.com/2017/02/27/la-compleja-realidadde-ser-gay-en-america-latina/

12. Butler, Judith. (2017). Marcos de guerra. Las vidas Iloradas. Barcelona: Paidós.

13. Camhaji, Elías. (2018, noviembre 17). El alcalde de Tijuana arremete contra la caravana de emigrantes. El País. https://elpais.com/internacional/2018/11/17/ mexico/1542412389_526379.html

14. Centro latino-americano em sexualidade e dereitos humanos (CLAM). (2011, septiembre 21). Mortalidad materna en América Latina. http://www.clam.org.br/ destaque/conteudo.asp? infoid $=8676$

15. Centro Nacional de Memoria Histórica (CNMH). (2011). Mujeres y guerra. Víctimas y resistentes en el Caribe colombiano. Bogotá, D. C.: CNMH.

16. Centro Nacional de Memoria Histórica (CNMH). (2015). Aniquilar la diferencia. Lesbianas, gays, bisexuales y transgeneristas en el marco del conflicto armado colombiano. Bogotá, D. C.: CNMH.

17. Comisión Interamericana de Derechos Humanos. (2015). Violencia contra personas lesbianas, gays, bisexuales, trans e intersex en América. Organización de los Estados Americanos. http://www.oas.org/es/cidh/informes/pdfs/ ViolenciaPersonasLGBTI.pdf 
El giro a la derecha en América Latina. La crueldad y el gobierno de los cuerpos-Otros...

18. Costa, Aurélio Antônio Ribeiro; Ribas, Maria do Socorro Sampaio de Sousa; Amorim, Melania Maria Ramos de e Santos, Luiz Carlos. (2002). Maternal Mortality in Recife. Revista Brasileira de Ginecologia e Obstetrícia, 24 (7), pp. 455-462. https:// doi.org/10.1590/S0100-72032002000700005

19. Dorlin, Elsa. (2008). Sexe, genre et sexualités: Introduction à la théorie féministe. Paris: PUF. https://doi.org/10.3917/puf.dorli.2008.01

20. Dorlin, Elsa. (2009). La matrice de la race généalogie sexuelle et coloniale de la Nation française. Paris: La Découverte.

21. El Desconcierto. (2018, noviembre 14). 137 propiedades fiscales a manos de iglesias evangélicas: Comunas más beneficiadas coinciden con alta votación de Kast. https://www.eldesconcierto.cl/nacional/2018/11/14/137-propiedades-fiscalesa-manos-de-iglesias-evangelicas-comunas-mas-beneficiadas-coinciden-con-altavotacion-de-kast.html

22. Estadão Conteúdo. (2018, octubre $\left.1^{\circ}\right)$. Bolsonaro recebe apoio de líderes evangélicos. Estado de Minas. https:/www.em.com.br/app/noticia/ politica/2018/10/01/interna_politica,993227/bolsonaro-recebe-apoio-de-lideresevangelicos.shtml

23. Evangélico Digital. (2018, septiembre 19). Gobierno de Argentina acude a las iglesias evangélicas para proveer ayuda social. https:/www.evangelicodigital.com/ latinoamerica/2596/gobierno-argentina-acude-a-iglesias

24. Fanon, Franz. (2009). Piel negra, máscaras blancas. Madrid: Akal. https://doi. org/10.7476/9788523212148

25. Federici, Silvia. (2018). El patriarcado del salario. Críticas feministas al marxismo. Madrid: Traficante de Sueños.

26. Foucault, Michel. (2007). Historia de la sexualidad 1. La voluntad del saber. Buenos Aires: Siglo xxı.

27. Foucault, Michel. (2008). Seguridad, territorio, población. Curso del Collège de France (1977-1978). Madrid: Akal.

28. France 24. (2018, octubre 21). El respaldo de los cristianos a Jair Bolsonaro. https://www.france24.com/es/20181021-elecciones-brasil-iglesia-evangelicabolsonaro

29. García Becerra, Andrea y Ojeda, Diana. (2018). Conjurar el olvido: Campesinos y política en las Ilanuras del Caribe colombiano en los años 70. Antípoda, 31, pp. 137-141. https://doi.org/10.7440/antipoda31.2018.07

30. Global Witness. (2018, julio 24). Deadliest Year on Record for Land and Environmental Defenders, as Agribusiness is Shown to be the Industry most Linked to Killings. https://www.globalwitness.org/en/press-releases/deadliest-year-record-landand-environmental-defenders-agribusiness-shown-be-industry-most-linked-killings/

31. Gómez Ponce de León, Rodolfo. (2016). Asistencia a mujeres en situación de aborto en America Latina y el Caribe [Presentación]. Organización Panamericana de la Salud. https://www.paho.org/clap/index.php?option=com_ 
docman \&view = download \&alias $=498$-aborto-inseguro\&category slug $=$ documentos-de-noticias\&ltemid $=219 \&$ lang $=$ en

32. Gómez-Sánchez, Pío Iván; Urquijo-Velásquez, Lenis Enrique y Villarreal, Cristina (2010). Estrategia FIGO para la prevención del aborto inseguro. Experiencia en Colombia. International Journal of Gynecology \& Obstetrics, 62 (1), pp. 24-35. https://doi.org/10.18597/rcog.228

33. Guttmacher Institute. (2018, marzo). Aborto en América Latina y el Caribe. https://www.guttmacher.org/sites/default/files/factsheet/fs-aww-lac-es.pdf

34. Harvey, David. (2007). El nuevo imperialismo. Madrid: Akal.

35. Harvey, David. (2008). El neoliberalismo como destrucción creativa. Apuntes del CENES, 27 (45), pp. 10-34.

36. Hidalgo, Martín. (2017, noviembre 12). La influencia evangélica en el Congreso. El Comercio. https://elcomercio.pe/politica/influencia-evangelica-congresonoticia-473036-noticia/

37. Horon, Isabelle \& Cheng, Diana. (2001). Enhanced Surveillance for PregnancyAssociated Mortality-Maryland, 1993-1998. JAMA, 285 (11), pp. 1455-1459. https:// doi.org/10.1001/jama.285.11.1455

38. Human Rights Watch. (2019, enero 17). Brazil: Bolsonaro Should Address Crime Lawfully. Troubling First Moves Include Plan to «Supervise» Independent Groups. https://www.hrw.org/news/2019/01/17/brazil-bolsonaro-should-addresscrime-lawfully

[140 ] 39. Human Rights Watch. (s. f.). Informe Mundial 2019. https://www.hrw.org/es/ world-report/2019\#

40. Hurtado, Paola; Gutiérrez, Jennifer; Gómez, Laura y Barbosa, Francy. (2019, marzo). Lideresas Sociales en Colombia: El relato invisible de la crueldad. CODHES. https://codhes.files.wordpress.com/2019/03/informe-lideresas-sociales-codhesmarzo-2019.pdf

41. Infobae. (2017, septiembre 5). Mauricio Macri destacó la presencia evangélica en el país y su batalla «por valores en los que creemos». https://www.infobae.com/ sociedad/2017/09/05/mauricio-macri-destaco-la-presencia-evangelica-en-el-pais-y-subatalla-por-valores-en-los-que-creemos/

42. La W Radio. (2018, mayo 9). Iván Duque logra importante apoyo en la comunidad cristiana. https://play.wradio.com.co/audio/3747582/

43. Lara, Carlos. (2017, febrero 16). Peña Nieto dialoga con líderes de Iglesias cristianas y evangélicas. El Sol de México. https://www.elsoldemexico.com.mx/ mexico/pena-nieto-dialoga-con-lideres-de-iglesias-cristianas-y-evangelicas-142396. html

44. Lugones, María. (2008). Colonialidad y género. Tabula Rasa, 9, pp. 73-102. https://doi.org/10.25058/20112742.340

45. Mantilla Falcón, Julissa. (2001). El caso de las esterilizaciones forzadas en el Perú como una violación de los derechos humanos. lus Et Veritas, 23, pp. 10-20. 
El giro a la derecha en América Latina. La crueldad y el gobierno de los cuerpos-Otros...

46. Mariano, Ricardo e Gerardi, Dirceu André. (2019). Eleições presidenciais na América Latina em 2018 e ativismo político de evangélicos conservadores. Revista USP, 120, pp. 61-76. https://doi.org/10.11606/issn.2316-9036.v0i120p61-76

47. Márquez Covarrubias, Humberto. (2017). Capitalismo del fin de los tiempos:actualidad de la simbiosis entre violencia y acumulación originaria. Estudios Críticos del Desarrollo, vil (13), pp. 189-253.

48. Mbembe, Achille. (2006). Nécropolitique. Raisons politiques, 21(1), pp. 2960. https://doi.org/10.3917/rai.021.0029

49. Moraña, Mabel y Sánchez Prado, Ignacio (eds.). (2012). El lenguaje de las emociones. Afecto y cultura en América Latina. Madrid: Iberoamericana. https://doi. org/10.31819/9783954870530

50. Mundo Cristiano. (2018, septiembre 18). Macri busca a iglesias evangélicas para lidiar con crisis económica que atraviesa Argentina. https://www1.cbn.com/ mundocristiano/latinoamerica/2018/september/macri-busca-a-iglesias-evangelicaspara-lidiar-con-crisis-economica-que-atraviesa-argentina

51. Nájar, Alberto. (2018, octubre 26). Enrique Peña Nieto: 5 razones que lo convirtieron en el presidente más impopular en la historia reciente de México. $B B C$ News Mundo. https://www.bbc.com/mundo/noticias-america-latina-46313984

52. Noguera, Carlos E. (2003). Medicina y política: Discurso médico y prácticas higiénicas durante la primera mitad del siglo xx en Colombia. Medellín: Universidad EAFIT.

53. Noticias. (2018, octubre 8). Influir en nombre de Dios: el crecimiento de los evangélicos en Argentina. https://noticias.perfil.com/noticias/general/2018-10-08influir-en-nombre-de-dios-el-crecimiento-de-los-evangelicos-en-argentina.phtml

54. Ochoa Muñoz, Karina. (2014). El debate sobre las y los amerindios: Entre el discurso de la bestialización, la feminización y la racialización. El Cotidiano, 184, pp. 13-22. http://revele.com.veywww.redalyc.org/articulo.oa?id=32530724005

55. Organización Mundial de la Salud (OMS). (2019, septiembre 19). Mortalidad materna. https://www.who.int/es/news-room/fact-sheets/detail/maternal-mortality

56. Oyěwùmí, Oyèrónk $\square$. (1997). The Invention of Women: Making an African Sense of Western Gender Discourses. Minneapolis: University of Minnesota.

57. Petteni, Oriane et Wustefeld, Sophie. (2016). Entretien avec Jules Falquet: Matérialisme féministe, crise du travail salarié et imbrication des rapports sociaux. Cahiers du GRM, 10. https://doi.org/10.4000/grm.839

58. Phillips, Dom. (2019, marzo 4). «We are fighting»: Brazil's indigenous groups unite to protect their land. The Guardian. https://www.theguardian.com/world/2019/ mar/04/we-are-fighting-brazils-indigenous-groups-unite-to-protect-their-land

59. Prakash, Neeti. (2019, abril 18). In Bolsonaro's Brazil, indigenous groups are struggling for basic human rights. Peoples Dispatch. https://peoplesdispatch. org/2019/04/18/in-bolsonaros-brazil-indigenous-groups-are-struggling-for-basichuman-rights/ 
60. Prensa Presidencial (2018, octubre 31). Presidente Piñera celebra el Día Nacional de las Iglesias Evangélicas y Protestantes. https://prensa.presidencia.cl/ discurso.aspx? $\mathrm{id}=85952$

61. Ruiz Tovar, Gonzalo. (2016, abril 12). Perú confirma un giro político hacia la derecha. El Comercio. https://www.elcomercio.com/actualidad/peru-eleccionesgiropolitico-derecha-fujimori.html

62. Saadawi, Nawal El. (2010). The Essential Nawal El Saadawi: A Reader. London, New York: Zed.

63. Saidel, Matías. (2018). Biopolítica y gubernamentalidad: Dos conceptos para problematizar el poder e interpretar el neoliberalismo. Ecopolítica, 21, pp. 17-37.

64. Salgado, Sebastián. (2018, octubre 17). Argentina ¿Macri negocia su reelección con la Iglesia Evangélica? Data Urgente. https://dataurgente.com/opinion/2018/10/17/ argentina-macri-negocia-reeleccion-con-la-iglesia-evangelica/

65. Sánchez, Gimena. (2019, febrero 7). At Least 13 Human Rights, Social Leaders Killed in Colombia So Far in 2019. WOLA. https://www.wola.org/2019/02/humanrights-social-leaders-killed-colombia-january-2019/

66. Sanchís, Ima. (2017, marzo 30). Nawal El Saadawi. Médica, escritora y activista: "La maternidad es una cárcel; el padre es libre pero la madre, no». El Clarín. https://www.clarin.com/cultura/dicen-eva-trajo-pecado-mundo-alguna-mujersiente_0_ryvXsxshx.html

\section{[142] Iv.v2i13.551}

67. Scott, Joan. (2001). Experiencia. La Ventana, 2 (13). https://doi.org/10.32870/

68. Segato, Rita. (2007). La nación y sus otros: raza, etnicidad y diversidad religiosa en tiempos de políticas de la identidad. Buenos Aires: Prometeo.

69. Segato, Rita. (2014). El sexo y la norma: frente estatal, patriarcado, desposesión, colonidad. Estudos Feministas, 22 (2), pp. 593-616. https://doi.org/10.1590/S0104026X2014000200012

70. Segato, Rita. (2015). La crítica de la colonialidad en ocho ensayos y una antropología por demanda. Buenos Aires: Prometeo.

71. Segato, Rita. (2016a). Género y colonialidad: en busca de claves de lectura y de un vocabulario estratégico descolonial. Núcleo de Identidades de Gênero e Subjetividades. https://doi.org/10.4000/eces.1533

72. Segato, Rita. (2016b). La guerra contra las mujeres. Madrid: Traficantes de Sueños.

73. Shaw, Dorothy. (2010). The FIGO Initiative for the Prevention of Unsafe Abortion. International Journal of Gynecology \& Obstetrics, 110, pp. 17-19. https:// doi.org/10.1016/j.ijgo.2010.04.004

74. Semana. (2019, julio 4). «Si la autoridad implica una masacre»: Uribe en contra de los acuerdos con la Minga. https://www.semana.com/nacion/articulo/ si-la-autoridad-implica-una-masacre-uribe-en-contra-de-los-acuerdos-con-laminga/608579/ 
75. Sieder, Rachel; Beltrán y Puga, Alma; Machado, Marta y Peñas Defago, Angélica. (2019). Derechos sexuales y reproductivos de las mujeres en América Latina a debate. Encartes, 2 (3), pp. 231-243. https://doi.org/10.29340/en.v2n3.99

76. Tauli-Corpuz, Victoria. (2018, agosto). Una carta de la Relatora Especial sobre los derechos de los pueblos indígenas. Universidad Nacional Autónoma de México. http://www.nacionmulticultural.unam.mx/mezinal/docs/9292.pdf

77. Valencia, Sayak. (2016). Capitalismo Gore. México, D. F.: Planeta.

78. Vargas, Daniela. (2019, marzo 14). Las razones de las movilizaciones indígenas en el Cauca. El Espectador. https://www.elespectador.com/noticias/ nacional/las-razones-de-las-movilizaciones-indigenas-en-el-cauca/

79. Villarraga Sarmiento, Álvaro (ed.). (2014). Región Caribe, Departamento de Antioquia, Departamento de Chocó. Bogotá, D. C.: CNMH.

80. Watson, Fiona. (2018, diciembre 31). The Uncontacted Tribes of Brazil Face Genocide Under Jair Bolsonaro. The Guardian. https://www.theguardian.com/ commentisfree/2018/dec/31/tribes-brazil-genocide-jair-bolsonaro

81. Watson, Fiona. (2019, abril 14). Bolsonaro: 100 días de guerra contra los pueblos indígenas. El País. https://elpais.com/elpais/2019/04/11/3500_ millones/1554971346 439815.html

82. Witker, Ivan. (2018, octubre 15). «Dios por encima de todos»: El irresistible ascenso evangélico en la política latinoamericana. El Mostrador. https://www. elmostrador.cl/noticias/opinion/columnas/2018/10/15/dios-por-encima-de-todos-elirresistible-ascenso-evangelico-en-la-politica-latinoamericana/ 